\title{
Approche multicritères pour le choix d'une installation de valorisation de déchets : application aux sédiments de l'écluse de Strasbourg (Rhin, France)
}

\section{Mickael BURONFOSSE ${ }^{1,2}$, Sokha LEANG ${ }^{1}$, Youcef BOUZIDI ${ }^{\text {, }}$ Nicolas ANTHEAUME ${ }^{3}$, François THERY ${ }^{1}$}

1. EDF R\&D, TREE, EDF Lab. Les Renardières, 77250, Moret-sur-Loing et Orvanne, France. mickael.buronfosse@edf.fr ; sokha.leang@edf.fr ; françois.thery@edf.fr

2. Université de Technologie de Troyes, CNRS, ICD - CREIDD, UMR 6281, 12 rue Marie Curie, CS 42060, 10 004, Troyes cedex, France. youcef.bouzidi@utt.fr

3. Université de Nantes, LEMNA, Chemin de la Censive du Tertre, BP 52231, 44322 Nantes Cedex 3, France. nicolas.antheaume@univ-nantes.fr

\section{Résumé :}

La gestion des déchets est complexe et doit pouvoir intégrer les volets (i) économique, (ii) environnemental et (iii) territorial en sus des traditionnels aspects (iv) technique et (v) réglementaire afin de s'insérer dans le concept de l'économie circulaire et le rendre opérationnel. La littérature scientifique montre que ces aspects sont rarement traités ensemble et il est difficile pour les décideurs opérationnels de choisir entre différentes installations de valorisation, en se basant sur une analyse complète. Forte de ce constat, la R\&D du groupe EDF travaille à l'élaboration d'un outil permettant de faciliter cet arbitrage. À partir de la méthodologie Analytic Hierarchy Process (AHP), vingt-deux critères qualitatifs et quantitatifs et l'avis d'une quarantaine d'experts (industriels, institutionnels et académiques), l'outil élaboré compare des installations de gestion de déchets en tenant compte des cinq dimensions citées précédemment. Dans cet article, nous proposons cette méthode de comparaison pour cinq voies de valorisation plausibles dans le cas d'une gestion à terre des sédiments du Rhin, dragués dans le domaine hydroélectrique concédé à EDF. Les performances des installations comparées varient selon les dimensions et l'outil donne la possibilité aux décideurs opérationnels de choisir, selon leur perception du contexte, une installation à partir du meilleur compromis entre les cinq aspects étudiés. Ainsi, le gestionnaire de sédiments et plus largement le gestionnaire de déchets peut argumenter une décision et dispose d'une base de discussion et de réflexion avec les différents services internes et externes concernés.

\section{Mots-clés :}

Aide à la décision multicritères, AHP, Valorisation des sédiments, Gestion des déchets. 


\section{Thème 6 - Gestion durable des zones littorales et estuariennes}

\section{Introduction}

Les industriels et les collectivités locales ont la responsabilité (WILSON, 2007) d'organiser l'orientation des flux de déchets vers les filières adéquates (JORF, 2015) en respectant les objectifs de valorisation européens (UE, 2008) et nationaux (JORF, 2015). À ce jour, les outils et méthodes principalement utilisés pour les études de gestion de déchets portent principalement sur les dimensions économique et environnementale (CHARISIOS et al., 2013 ; ALLESCH \& BRUNNER, 2014) alors que les dimensions technique (NOURI et al., 2018 ; DEBRAY, 1997), réglementaire et territoriale doivent, également, être analysées (CHARISIOS et al., 2013 ; MARCOUX et al., 2016). La littérature scientifique montre que ces aspects sont rarement traités ensemble et il est difficile pour les décideurs opérationnels de choisir entre différentes installations de valorisation, en se basant sur une analyse complète (ROJO, 2009). En conséquence, il est difficile pour les décideurs opérationnels de choisir entre différentes installations de valorisation. Pour faciliter cet arbitrage, la $R \& D$ du Groupe EDF propose d'aider le décideur opérationnel à faire un choix pour gérer ses déchets en évaluant les performances de différentes installations de gestion selon les cinq dimensions citées.

Cet article présente la mise au point d'un outil multicritère d'aide à la décision à partir de la méthodologie Analytic Hierarchy Process (AHP) (SAATY, 1977) et son application.

\section{Démarche mise en œuvre}

Nous présentons ici la démarche globale "Connaitre - Localiser - Evaluer" étudiée par la R\&D d'EDF et plus précisément la partie "Evaluer" appliquée au cas de la valorisation de sédiments.

\subsection{L'outil CLE (Connaître, Localiser, Evaluer)}

La R\&D du groupe EDF a mis au point une approche permettant d'identifier et d'étudier les installations pouvant prendre en charge le traitement et la valorisation des déchets (Figure 1).

Cette approche se décline en trois grandes étapes. La première permet de "Connaître » les caractéristiques et les critères d'acceptation des différentes filières de gestion (THERY, 2013). Cette adéquation technique au niveau des filières doit souvent correspondre à une adéquation géographique pour que la solution soit rentable. Ainsi, des bases de données doivent être construite et couplées (ou via l'outil interne RECYTER) pour identifier et "Localiser" des installations de valorisation au niveau d'un territoire. Enfin, pour "Évaluer" et comparer plusieurs solutions (installations) de valorisation, il est important de prendre en compte les dimensions citées précédemment. Ici, nous développons et discutons la partie "Evaluer" de la démarche. 


\section{XVèmes Journées Nationales Génie Côtier - Génie Civil \\ La Rochelle, 29 au 31 mai 2018}

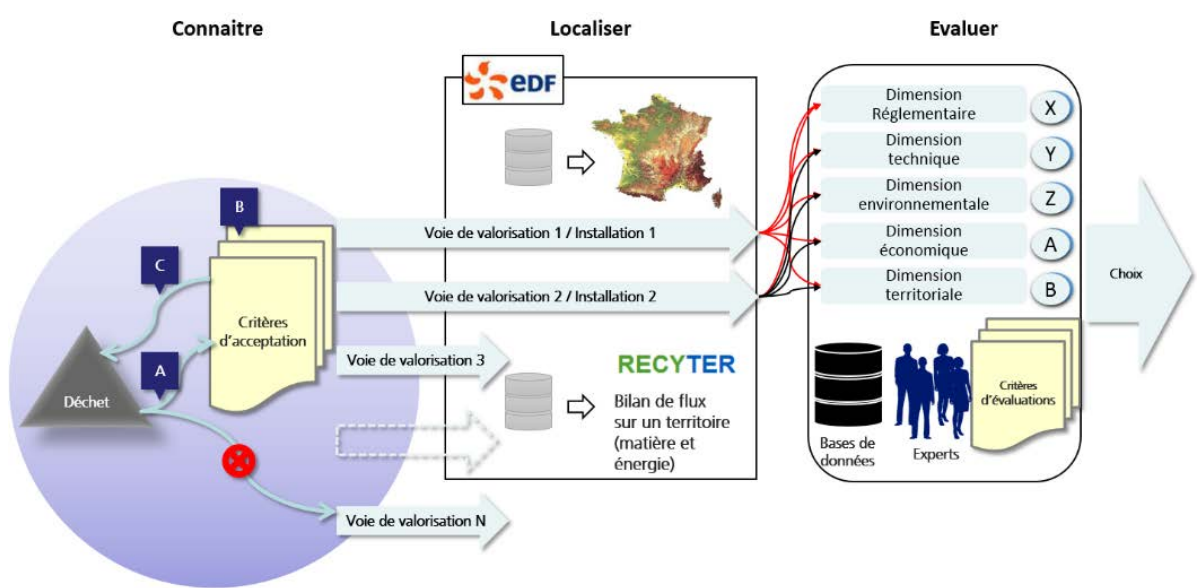

Figure 1. Démarche intégrée d'EDF R\&D.

\subsection{Développement de la partie Evaluer}

Celle-ci propose de comparer les installations de gestion de déchets à partir de vingtdeux critères qualitatifs et quantitatifs (tableau 1) et l'avis d'une quarantaine d'experts des milieux industriels, institutionnels et académiques.

Tableau 1. Liste des critères retenus par dimension.

\begin{tabular}{|c|c|c|c|c|}
\hline Réglementaire & Économique & Technique & Environnemental & Territorial \\
\hline $\begin{array}{l}R_{1} \text { Respect de la } \\
\text { hiérarchie de la } \\
\text { gestion des } \\
\text { déchets }\end{array}$ & $\begin{array}{l}E c_{1} \text { Cout } \\
\text { d'utilisation de la } \\
\text { filière }\end{array}$ & $\begin{array}{l}T_{1} \text { Pérennité de la } \\
\text { filière }\end{array}$ & $\begin{array}{l}\text { Env }{ }_{1} \text { Absence } \\
\text { d'impacts } \\
\text { environnementaux }\end{array}$ & $\begin{array}{l}\text { Ter }_{1} \text { Favoriser } \\
\text { l'économie locale }\end{array}$ \\
\hline $\begin{array}{l}R_{2} \text { Niveau de } \\
\text { responsabilité }\end{array}$ & $\begin{array}{l}\text { Ec } c_{2} \text { Intensité } \\
\text { concurrentielle }\end{array}$ & $\begin{array}{l}T_{2} L \text { 'adéquation } \\
\text { du déchet en } \\
\text { entrée de filière }\end{array}$ & $\begin{array}{l}\text { Env } v_{2} \text { Changement } \\
\text { climatique }\end{array}$ & $\begin{array}{l}\mathrm{Ter}_{2} \text { Utilisation } \\
\text { locale de la } \\
\text { ressource }\end{array}$ \\
\hline $\begin{array}{l}R_{3} \text { Respect du } \\
\text { principe de } \\
\text { proximité }\end{array}$ & $\begin{array}{l}E c_{3} \text { Intensité de la } \\
\text { demande }\end{array}$ & $\begin{array}{l}T_{3} L \text { 'adéquation } \\
\text { du produit dans } \\
\text { une filière }\end{array}$ & $\begin{array}{l}\text { Env }_{3} \text { Extraction de } \\
\text { matières premières }\end{array}$ & $\begin{array}{l}\mathrm{Ter}_{3} \text { Capacité à } \\
\text { transporter le } \\
\text { déchet sur le } \\
\text { territoire }\end{array}$ \\
\hline \multirow[t]{3}{*}{$\begin{array}{l}R_{4} \text { Positionnement } \\
\text { de la gestion vis- } \\
\grave{a} \text {-vis des objectifs } \\
\text { de valorisation }\end{array}$} & & $\begin{array}{l}T_{4} \text { Multiplicité des } \\
\text { produits }\end{array}$ & $\begin{array}{l}\text { Env }{ }_{4} \text { Consommation } \\
\text { en eau }\end{array}$ & $\mathrm{Ter}_{4}$ Emploi \\
\hline & & $\begin{array}{l}T_{5} \text { Capacité } \\
\text { d'absorption } \\
\text { annuelle de } \\
\text { l'installation }\end{array}$ & $\begin{array}{l}\text { Env } v_{5} \text { Consommation } \\
\text { en énergie }\end{array}$ & $\begin{array}{l}\text { Ter }_{5} \text { Incitations } \\
\text { locales }\end{array}$ \\
\hline & & $\begin{array}{l}T_{6} \text { Flexibilité } \\
\text { d'absorption de } \\
\text { l'installation }\end{array}$ & & \\
\hline
\end{tabular}

Pour construire et pondérer les critères entre eux, nous avons choisi d'adapter la méthodologie Analytic Hierarchy Process (AHP) (SAATY, 1977 ; BURONFOSSE et al., 2017). Plusieurs analyses, effectuées sur différents types de déchets de bois 


\section{Thème 6 - Gestion durable des zones littorales et estuariennes}

(BURONFOSSE et al., 2018) et de déchets amiantés montrent le large spectre d'application de l'outil.

\subsection{Présentation du cas d'étude : gestion à terre des sédiments du Rhin}

Nous proposons, ici, de l'appliquer au cas des sédiments de l'écluse de Strasbourg. Le groupe EDF exploite une large part des installations du parc hydraulique français et doit gérer des sédiments dragués. Au niveau de l'écluse de Strasbourg, la première extraction de 12500 tonnes de sédiments, considérés comme inertes, conforte la R\&D d'EDF à renforcer la recherche de voies de valorisation. Parmi les solutions de gestion concevables pour les sédiments de l'écluse de Strasbourg, cinq possibilités sont retenues : $\mathrm{V}_{1}$-Construction de sol, $\mathrm{V}_{2}$-Valorisation en technique routière, $\mathrm{V}_{3}$-Valorisation en cimenterie, $\mathrm{V}_{4}$-Elimination en Slufter (zone de dépôt destinée à stocker des boues) à Rotterdam et $\mathrm{V}_{5}$-Elimination en installation de stockage de déchets inertes. En effet, des études dédiées menées, récemment, sur la valorisation des sédiments des ouvrages hydroélectriques ont montré que les filières de valorisation $V_{1}$ à $V_{3}$ peuvent accepter une intégration de sédiments en substitution de matières premières (ANGER et al., 2014 ; FAURE et al., 2017 ; FOURVEL et al., 2018). Les gestionnaires de sédiments du site sont alors confrontés au questionnement suivant :

"Comment arbitrer entre ces différentes possibilités de gestion pour le devenir des sédiments de l'écluse de Strasbourg ?".

\section{Résultats et discussion}

3.1 Comparaison globale des solutions de gestion envisageable

Le premier résultat préliminaire consiste à identifier et à localiser les installations correspondantes aux voies de gestion citées précédemment (tableau 2) :

Tableau 2. Identification et localisation des installations de gestion de déchets.

\begin{tabular}{|c|c|c|c|c|}
\hline $\mathrm{V}_{1}$ & $\mathrm{~V}_{2}$ & $\mathrm{~V}_{3}$ & $\mathrm{~V}_{4}$ & $\mathrm{~V}_{5}$ \\
\hline Construction sol & Colas (67) & Heming (57) & Slufter (Rotterdam) & Suez/Sita (67) \\
\hline$* \mathrm{Hyp}: 50 \mathrm{~km}$ & $7 \mathrm{~km}$ & $92 \mathrm{~km}$ & $660 \mathrm{~km}$ & $8 \mathrm{~km}$ \\
\hline
\end{tabular}

Note: *Nous n'avons pas identifié d'installation précise. Nous avons pris l'hypothèse qu'il en existe une dans les 50 kilomètres.

Ensuite, pour utiliser la totalité des critères, il est nécessaire de disposer d'un jeu de données associées à chaque installation. Ce dernier est écrit dans BURONFOSSE et al. (2017). L'histogramme ci-dessous (figure 2) montre les résultats globaux par dimension des cinq installations comparées. 


\section{XVèmes Journées Nationales Génie Côtier - Génie Civil \\ La Rochelle, 29 au 31 mai 2018}

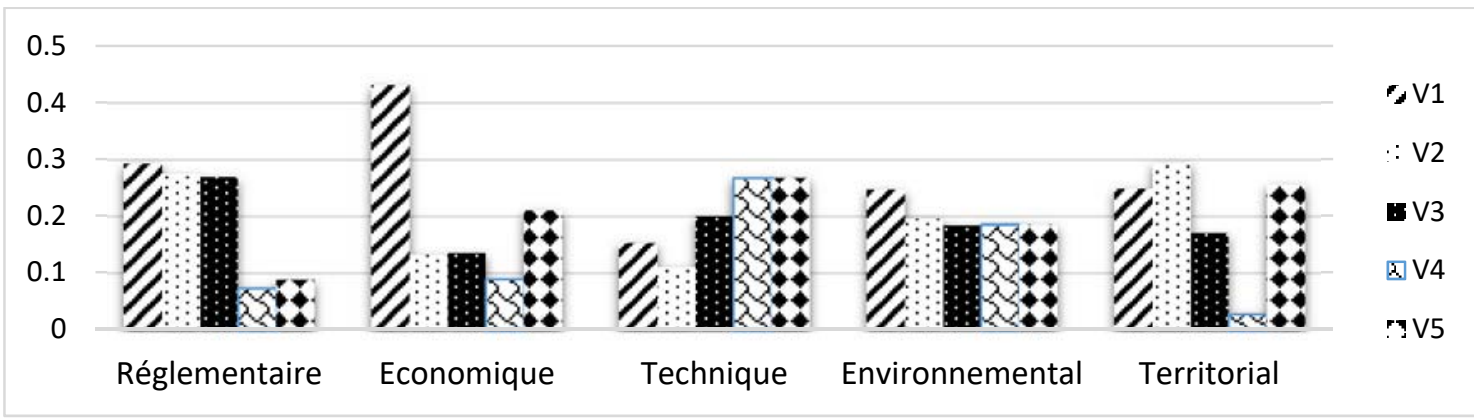

Figure 2. Résultats de la comparaison des installations sur les 5 dimensions.

Nous remarquons aisément qu'il n'y a pas une solution idéale, puisque chacune des installations a ses forces et ses faiblesses sur chacune des dimensions. En effet, il en ressort qu'il est préférable de diriger le flux de sédiments vers les installations de valorisation $\mathrm{V}_{1}, \mathrm{~V}_{2}$ et $\mathrm{V}_{3}$ d'un point de vue réglementaire, mais plutôt vers les installations $V_{3}, V_{4}$ et $V_{5}$ pour des aspects techniques. En revanche, il est plus avisé de valoriser les sédiments vers l'installation de valorisation en construction de sol $\left(\mathrm{V}_{1}\right)$ pour des aspects économiques et environnementaux. Pour finir, il est évident que l'installation $\mathrm{V}_{4}$ ne permet pas de répondre aux enjeux de la dimension territoriale. Nous avons aussi travaillé la représentation graphique des résultats afin de faciliter leur mise en visibilité (figure3).

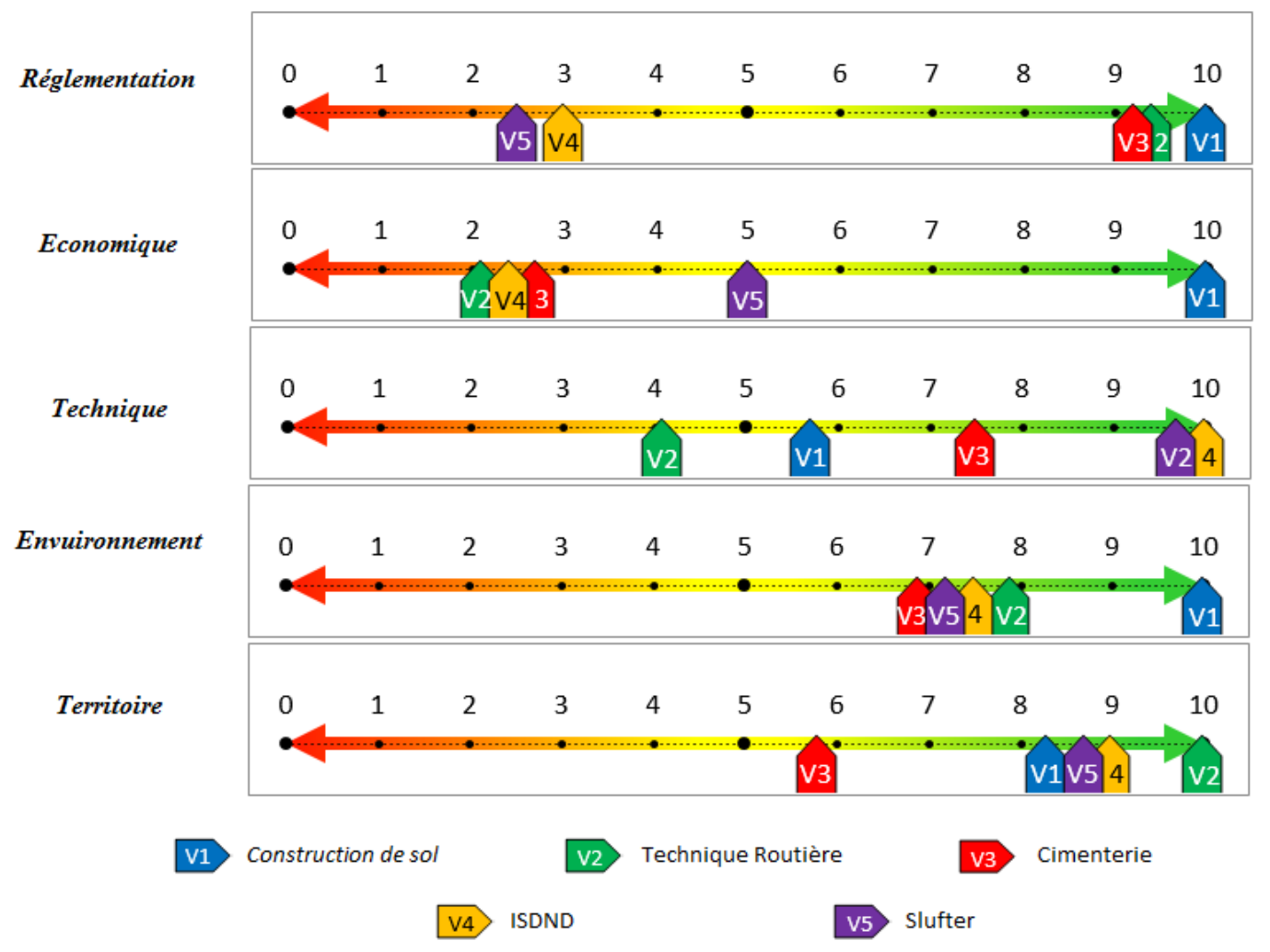

Figure 3. Résultats de la comparaison des installations sur les 5 dimensions. 


\section{Thème 6 - Gestion durable des zones littorales et estuariennes}

Ces résultats globaux permettent une première approche, mais ne doivent pas occulter une analyse approfondie par dimension. En effet, une analyse complète est nécessaire pour repérer les critères importants intervenus dans le calcul de la note globale, de vérifier si ces mêmes critères sont définis par un poids fort ou non et d'analyser la sensibilité des résultats en fonction du jeu de données ou des hypothèses choisies.

\subsection{Comparaison détaillée des solutions de gestion envisageable}

L'outil développé permet une discussion approfondie des résultats. Celle-ci est possible grâce à la transparence de la méthode d'aide à la décision multicritères AHP et de l'ensemble du modèle. À titre d'exemple, la figure 4 ci-dessous montre les résultats détaillés de la comparaison des installations sur la dimension réglementaire, composée de quatre critères d'évaluation à savoir $\mathrm{R}_{1}$ - Respect de la hiérarchie, $\mathrm{R}_{2}$ - Niveau de la responsabilité, $\mathrm{R}_{3}$ - Respect du principe de proximité et $\mathrm{R}_{4}$ - Respect des objectifs de valorisation de l'entreprise.

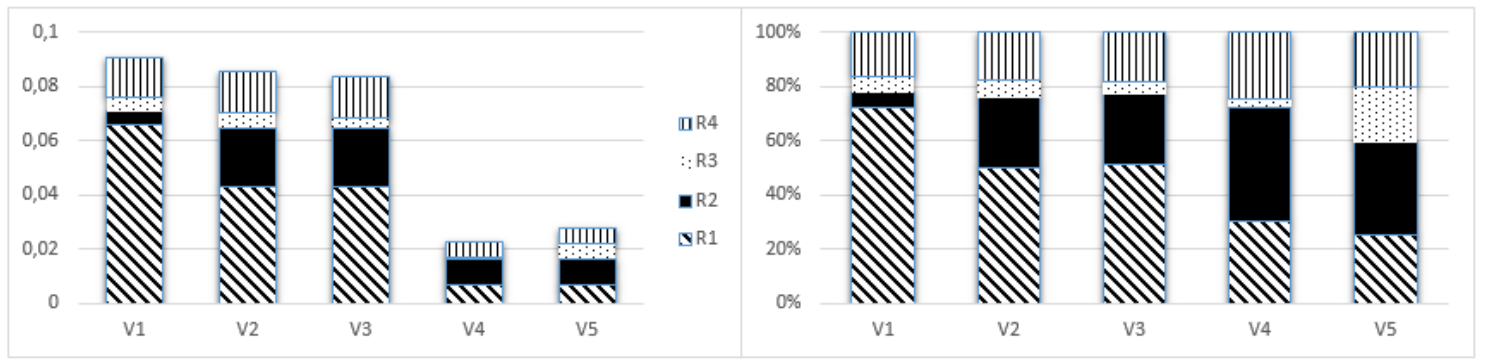

Figure 4. Résultats détaillés de la comparaison sur la dimension réglementaire.

L'histogramme de gauche permet de visualiser les résultats bruts de la comparaison, où l'on remarque clairement la dominance de l'installation $\mathrm{V}_{1}$ construction de sol, puis des deux installations de valorisation $V_{2}$ technique routière et $V_{3}$ cimenterie. L'histogramme empilé (côté droit de la figure 4) permet de visualiser l'importance de la performance de l'installation sur chacun des critères par rapport à la note finale. Cette représentation permet, par exemple, de comprendre que le résultat du critère $\mathrm{R}_{1}$ - Respect de la hiérarchie de la gestion des déchets contribue fortement à la note réglementaire des installations $\mathrm{V}_{1}$ à $\mathrm{V}_{3}$. Le second critère qui donne la priorité réglementaire à ces installations est $\mathrm{R}_{4}$-Positionnement de la gestion vis-à-vis des objectifs de valorisation. À la fin de la démarche, les gestionnaires de sédiments peuvent alors choisir, selon leur perception du contexte, une installation à partir du meilleur compromis entre les cinq dimensions.

\section{Conclusion}

En proposant, sur la base des recommandations d'experts, un ordre d'importance parmi les critères à prendre en considération, la méthode d'évaluation développée permet 


\section{XVèmes Journées Nationales Génie Côtier - Génie Civil \\ La Rochelle, 29 au 31 mai 2018}

d'éclairer et d'appuyer la prise de décision du gestionnaire de sédiments et plus largement du gestionnaire de déchets. Pour fiabiliser et comprendre le bilan de la comparaison, le modèle donne la possibilité d'effectuer une analyse approfondie des résultats en fonction des données et des hypothèses retenues, afin de discuter de leurs influences sur les résultats finaux. Toutefois, il ne propose pas la meilleure des solutions comparées ou la solution idéale, puisque les cinq dimensions ne sont pas agrégées. L'outil laisse le choix aux décideurs opérationnels, en leur donnant la possibilité de choisir une voie de valorisation selon le meilleur compromis et l'expérience du contexte locale qu'ils possèdent. Cet outil d'aide à la gestion des déchets permet, également, d'appuyer une décision et d'avoir une base de discussion et de réflexion avec les différents services internes et externes concernés pour les 5 dimensions cités dans la partie introduction.

L'outil développé (sur la base de la méthode AHP) offre adaptabilité et modularité. Il apporte une aide à la décision pour le choix d'une installation de valorisation de déchets dans un environnement multicritère.

\section{Références bibliographiques}

ALLESCH A., BRUNNER P.H. (2014). Assessment methods for solid waste management: A literature review. Waste Management \& Research, Vol. 32(6), pp 461-473. https://doi.org/10.1177/0734242X14535653

ANGER B., LEVACHER D., MANGWAL N., THERY F. (2014). Démarche d'identification et valorisation des sédiments de barrage en matériaux routiers. XIIIèmes Journées Nationales Génie Côtier - Génie Civil, Dunkerque, 2-4 juillet 2014, Editions Paralia, pp 941-952. https://doi.org/10.5150/ingcgc.2014.104

BURONFOSSE M., LEANG S., BOUZIDI Y., ANTHEAUME N., THERY F. (2017). Selection of use channels for dredged sediments: a multicriteria analysis approach. Coastal and Maritime Mediterranean Conference, Split 2017, Editions Paralia, pp 2732. https://doi.org $/ 10.5150 / \mathrm{cmcm} .2017 .006$

BURONFOSSE M., ANTHEAUME N., LEANG S., BOUZIDI Y., THERY F. (2018). Construction d'un outil de gestion environnementale. Cas de la gestion des déchets. 39e congrès de l'AFC, Nantes 2018, (in press)

CHARISIOS A., MOUSSIOPOULOS N., KARAGIANNIDIS A., BANIAS G., PERKOULIDIS G. (2013). The use of multi-criteria decision analysis to tackle waste management problems: a literature review. Waste Management \& Research, Vol. 31 (2), pp 115-129. https://doi.org/10.1177/0734242X12470203

DEBRAY B. (1997). Systèmes d'aide à la décision pour le traitement des déchets industriels spéciaux. INSA de Lyon. URL: https://tel.archives-ouvertes.fr/tel-00850534/

FAURE A., SMITH A., COUDRAY C., ANGER B., COLINA H., MOULIN I., THERY F. (2017). Ability of two dam fine-grained sediments to be used in cement industry as raw material for clinker production and as pozzolanic additional constituent 


\section{Thème 6 - Gestion durable des zones littorales et estuariennes}

of Portland-composite cement. Waste and Biomass Valorization, Vol. 8(6), pp 21412163. https://doi.org/10.1007/s12649-017-9870-8

FOURVEL G., VIDAL-BEAUDET L., LE BOCQ A., BROCHIER V., THÉRY F., LANDRY D., KUMARASAMY T., CANNAVO P. (2018). Early structural stability of fine dam sediment in soil construction. Journal of Soils and Sediments, in press, 1-17. https://doi.org/10.1007/s11368-018-1926-2

JORF. (2015). Loi $n^{\circ}$ 2015-992 du 17 août 2015 relative à la transition énergétique pour la croissance verte. pp 2015-992.

MARCOUX M.-A., OLIVIER F., THERY F., RESEAU COOPERATIF DE RECHERCHES SUR LES DECHETS (FRANCE). (2016). Déchets et économie circulaire : conditions d'intégration pour une valorisation en filières industrielles. Editions Lavoisier : Tec \& Doc, 368 p.

NOURI D., SABOUR M.R., GHANBARZADEHLAK M. (2018). Industrial solid waste management through the application of multi-criteria decision-making analysis: A case study of Shamsabad industrial complexes. Journal of Material Cycles and Waste Management, Vol. 20(1), pp 43-58. https://doi.org/10.1007/s10163-016-0544-6

ROJO G. (2009). La gestion dynamique des déchets (GDD) : élaboration d'une approche intégrée d'aide à la décision visant à soutenir une gestion systémique et évolutive des déchets. Thèse de doctorat, Ecole Nationale Supérieure des Mines de Saint-Etienne. URL : https://tel.archives-ouvertes.fr/tel-00772106/document

SAATY T.L. (1977). A scaling method for priorities in hierarchical structures. Journal of Mathematical Psychology. Vol. 15(3), pp 234-281. https://doi.org/10.1016/0022-2496(77)90033-5

THERY F. (2013). Caractérisation des déchets en réponse aux critères d'acceptation des filières de valorisation: la clé du management vue par le producteur. Environnement \& technique, 2013-03-01, n 323, pp 55-60.

UE (2008). Directive 2008/98/EC of the European Parliament and of the Council on waste and repealing certain Directives. Brussels.

WILSON D.C. (2007). Development drivers for waste management. Waste Management \& Research, Vol. 25(3), pp 198-207. https://doi.org/10.1177/0734242X07079149 